\title{
CRITERIA FOR A BLASCHKE QUOTIENT TO BE OF UNIFORMLY BOUNDED CHARACTERISTIC
}

\author{
SHINJI YAMASHITA
}

ABstract. Criteria for a quotient $B_{1} / B_{2}$ of Blaschke products $B_{1}$ and $B_{2}$ to be of uniformly bounded characteristic are proposed in terms of interpolating sequences.

A Blaschke product is a holomorphic function

$$
B\left(z ;\left\{a_{n}\right\}\right)=\prod_{n=1}^{\infty}\left(\frac{\left|a_{n}\right|}{a_{n}}\right) \frac{a_{n}-z}{1-\bar{a}_{n} z}
$$

in the disk $D=\{|z|<1\}$, where $\left\{a_{n}\right\}$ is a sequence of complex numbers in $D$ with $\sum\left(1-\left|a_{n}\right|\right)<\infty$, with the convention $\left|a_{n}\right| / a_{n}=1$ for $a_{n}=0$. A Blaschke quotient is a meromorphic function $B_{1} / B_{2}$, where $B_{1}$ and $B_{2}$ are Blaschke products with no common zero. J. A. Cima and P. Colwell [2, Theorem 2] established a criterion for $B_{1} / B_{2}$ to be normal in $D$ in the sense of O. Lehto and K. I. Virtanen [4] in terms of interpolating sequence in the sense of L. Carleson [1]. Here a function $f$ meromorphic in $D$ is normal if $\left(1-|z|^{2}\right) f^{\#}(z)$ is bounded where, $f^{\#}=\left|f^{\prime}\right| /\left(1+|f|^{2}\right)$, and a sequence of points $\left\{z_{n}\right\}$ in $D$ is interpolating (or uniformly separated [3, p. 148]) in $D$ if

$$
\inf _{n \geqslant 1} \prod_{\substack{k=1 \\ k \neq n}}^{\infty}\left|\frac{z_{k}-z_{n}}{1-\bar{z}_{k} z_{n}}\right|>0
$$

If $\left\{z_{n}\right\}$ is interpolating in $D$, then $\Sigma\left(1-\left|z_{n}\right|\right)<\infty$. Cima and Colwell's cited result is (I) $\Leftrightarrow$ (II) in

THEOREM. Let $\left\{a_{n}^{(1)}\right\}$ and $\left\{a_{n}^{(2)}\right\}$ be disjoint interpolating sequences of points in $D$, and set

$$
B_{k}(z)=B\left(z ;\left\{a_{n}^{(k)}\right\}\right), \quad k=1,2 .
$$

Then the following are mutually equivalent.

(I) $B_{1} / B_{2}$ is normal in $D$.

(II) The sequence $\left\{a_{n}^{(1)}\right\} \cup\left\{a_{n}^{(2)}\right\}$ is interpolating in $D$.

(III) $B_{1} / B_{2}$ is of uniformly bounded characteristic in $D$.

Received by the editors February 18, 1984 and, in revised form, July 11, 1984.

1980 Mathematics Subject Classification. Primary 30D45, 30D50.

Key words and phrases. Blaschke product, interpolating sequence, normal meromorphic function, function of uniformly bounded characteristic.

(C1985 American Mathematical Society $0002-9939 / 85 \$ 1.00+\$ .25$ per page 
To explain the terminology we set $f_{w}(z)=f((z+w) /(1+\bar{w} z)), z, w \in D$, for $f$ meromorphic in $D$. We call $f$, of uniformly bounded characteristic in $D, f \in \mathrm{UBC}$ for short, if

$$
T\left(1, f_{w}\right)=\lim _{r \rightarrow 1-0} T\left(r, f_{w}\right), \quad w \in D
$$

is bounded in $D$, where

$$
T\left(r, f_{w}\right)=\int_{0}^{r}(\pi t)^{-1}\left[\iint_{|z|<t}\left(f_{w}\right)^{\#}(z)^{2} d x d y\right] d t
$$

is the Shimizu-Ahlfors characteristic function of $f_{w}$; see [5]. Since $f=f_{0}$, each $f \in \mathrm{UBC}$ is of bounded (Nevanlinna) characteristic in $D$.

Since each $f \in \mathrm{UBC}$ is normal in $D$ by [5, Theorem 3.1, p. 383], (III) $\Rightarrow$ (I) is obvious. To establish our Theorem the remaining work is

Proof OF (II) $\Rightarrow$ (III). First we observe, for $f=B_{1} / B_{2}$, the identity

$$
T\left(1, f_{w}\right)=\hat{F}(w)-F(w), \quad w \in D,
$$

where $F=\frac{1}{2} \log \left(\left|B_{1}\right|^{2}+\left|B_{2}\right|^{2}\right)$ and $\hat{F^{\wedge}}$ is the least harmonic majorant of the subharmonic function $F$ in $D$. For the proof of (1) we fix $w \in D$, and we let

$$
D(w, r)=\{\zeta ;|(\zeta-w) /(1-\bar{w} \zeta)|<r\}, \quad 0<r<1 .
$$

Then the Green function of $D(w, r)$ with its pole at $w$ is

$$
g_{r}(\zeta)=\log |r(1-\bar{w} \zeta) /(\zeta-w)|, \quad \zeta \in D(w, r) .
$$

Since the Laplacian $\Delta F=2 f^{\# 2}$ in $D$, the change of variable $z=(\zeta-w) /(1-\bar{w} \zeta)$, $\zeta \in D(w, r)$, together with

$$
T\left(r, f_{w}\right)=\pi^{-1} \iint_{|z|<r}\left(f_{w}\right)^{\#}(z)^{2} \log |r / z| d x d y,
$$

derived from [5, (2.5), p. 352] for $f_{w}$, yields

$$
T\left(r, f_{w}\right)=(2 \pi)^{-1} \iint_{D(w, r)}(\Delta F(\zeta)) g_{r}(\zeta) d \xi d \eta
$$

The Green formula

$$
\iint_{G}(\phi \Delta \psi-\psi \Delta \phi) d x d y=\int_{\partial G}\left(\psi \frac{\partial \phi}{\partial \nu}-\phi \frac{\partial \psi}{\partial \nu}\right) d s
$$

for $G=D(w, r) \backslash D(w, \varepsilon)(0<\varepsilon<r), \phi=g_{r}, \psi=(2 \pi)^{-1} F$, in the limiting case $\varepsilon \downarrow 0$, then reads

$$
T\left(r, f_{w}\right)=\hat{F_{r}}(w)-F(w),
$$

where $\hat{F_{r}}$ is the least harmonic majorant of $F$ in $D(w, r)$; to be more precise,

$$
\hat{F_{r}}(w)=(2 \pi)^{-1} \int_{\partial D(w, r)} F(\zeta) \frac{\partial}{\partial \nu} g_{r}(\zeta) d s,
$$


where $\partial / \partial \nu$ denotes the derivative in the inward-normal direction and $d s$ is the element of arc length. Letting $r \uparrow 1$ in (2) we obtain (1).

Suppose (II), and suppose then that

$$
\inf _{z \in D} F(z)=-\infty \text {. }
$$

Then there exists a sequence $\left\{z_{n}\right\}$ in $D$ such that

$$
\left|B_{1}\left(z_{n}\right)\right|^{2}+\left|B_{2}\left(z_{n}\right)\right|^{2} \rightarrow 0 \text {. }
$$

By the same argument as in [2, p. 798], this contradicts our hypothesis (II). Therefore $F$ is bounded from below and above in $D:-\infty<m \leqslant F \leqslant \log \sqrt{2}$. Thus,

$$
F^{\wedge}-F \leqslant \log \sqrt{2}-m \text { in } D,
$$

whence $f \in \mathrm{UBC}$ by (1).

REMARK. It follows from (II) that

$$
\inf _{z \in D}\left(\left|B_{1}(z)\right|^{2}+\left|B_{2}(z)\right|^{2}\right)>0 \text {. }
$$

Our proof shows that (IV) $\Rightarrow$ (III).

\section{REFERENCES}

1. Lennart Carleson, An interpolation problem for bounded analytic functions, Amer. J. Math. 80 (1958), 921-930.

2. Joseph A. Cima and Peter Colwell, Blaschke quotients and normality, Proc. Amer. Math. Soc. 19 (1968), 796-798.

3. Peter L. Duren, Theory of $H^{p}$ spaces, Academic Press, New York, 1970.

4. Olli Lehto and Kaarlo I. Virtanen, Boundary hehaviour and normal meromorphic functions, Acta Math. 97 (1957), 47-65.

5. Shinji Yamashita, Functions of uniformly bounded characteristic, Ann. Acad. Sci. Fenn. Ser. A I Math. 7 (1982), 349-367.

Department of Mathematics, Tokyo Metropolitan University, Fukasawa, Setagaya, Tokyo 158, JAPAN 